\title{
Supporting Information\
}

Manuscript: ac071003y

Title: Electrokinetic Fluid Control in Two-Dimensional Planar Microfluidic Devices

Authors: Margaret A. Lerch and Stephen C. Jacobson

\section{Microfluidic Device Fabrication}

PDMS/Glass Devices. Standard photolithography was used to create SU-8 masters from which the PDMS substrates with channels were cast. ${ }^{1}$ Masters were formed on $50 \mathrm{~mm} \times 50 \mathrm{~mm}$ glass substrates, which were cleaned with methanol prior to use. The substrates were first coated with AP300 Adhesion Promoter (Silicon Resources, Inc., Chandler, AZ). To further increase the lifetime of the master, a 20- $\mu \mathrm{m}$ base layer of SU-8 2010 (MicroChem Corp., Newton, MA) was spin-coated onto the substrate immediately after the AP300. A second SU-8 layer, also $20 \mu \mathrm{m}$ thick, was used to create the channel structures. Both layers were processed identically, except the first layer was exposed to UV light without a photomask. For photoresist application, the spin cycle was $500 \mathrm{rpm}$ for $5 \mathrm{~s}$ and ramped at $300 \mathrm{rpm} / \mathrm{s}$ to $1000 \mathrm{rpm}$ for $30 \mathrm{~s}$. After prebaking on a hot plate for $1 \mathrm{~min}$ at $65^{\circ} \mathrm{C}$ and $3 \mathrm{~min}$ at $95^{\circ} \mathrm{C}$, the photoresist was exposed to $270 \mathrm{~mJ} / \mathrm{cm}^{2}$ of UV light from an exposure system (205S, Optical Associates, Inc., San Jose, CA). The channel patterns were transferred to the photoresist from photomasks drawn in AutoCAD 2004 LT (AutoDesk, Inc., San Rafael, CA) and printed on a transparency using a high-resolution laser printer at 8000 dpi (Laserlab, Inc., San Diego, CA) or 40,460 dpi (Photoplot Store, Colorado Springs, CO). After exposure, the photoresist-coated substrate was baked using the same conditions as prior to exposure. The masters were developed for $2 \mathrm{~min}$ in SU-8 Developer (MicroChem Corp.), rinsed with 2-propanol, and dried with nitrogen. The channel heights of 
SU-8 masters were measured with a stylus profiler (Dektak 6M, Veeco Instruments, Inc., Woodbury, NY) and are listed in Table S1.

Table S1. Channel Heights for SU-8 Masters.

\begin{tabular}{|c|c|c|c|c|}
\hline $\begin{array}{c}\text { Channel } \\
\text { Design }\end{array}$ & $\begin{array}{c}\text { Planar region } \\
\text { width }(\mathrm{mm})\end{array}$ & $\begin{array}{c}\text { Number of } \\
\text { control channels }\end{array}$ & $\begin{array}{c}\text { Channel } \\
\text { height }(\mu \mathrm{m})\end{array}$ & $\begin{array}{c}\text { Standard } \\
\text { deviation, } \sigma(\mu \mathrm{m})\end{array}$ \\
\hline \hline Open & 2 & 1 & 21.2 & 0.06 \\
\hline Open & 2 & 2 & 19.5 & 0.3 \\
\hline Open & 2 & 4 & 18.8 & 0.2 \\
\hline Open & 1.55 & 4 & 20.7 & 0.2 \\
\hline Parallel & 1.55 & 4 & 20.7 & 0.3 \\
\hline
\end{tabular}

The PDMS silicone elastomer kit (Sylgard 184, Dow Corning Corp., Midland, MI) contained a polymer base and curing agent that were mixed in a 10:1 ratio. A tape barrier was placed around the mold to contain the elastomer mixture after being poured onto the master. The PDMS on the mold was placed under low vacuum ( $\sim 1$ Torr) for $1 \mathrm{~h}$ to enhance channel replication and was cured by heating at either $70^{\circ} \mathrm{C}$ for $1 \mathrm{~h}$ in an oven or $120^{\circ} \mathrm{C}$ for $20 \mathrm{~min}$ on a hotplate. The PDMS substrate was then separated from the master, and reservoirs were punched into the elastomer with a $3 \mathrm{~mm}$ cork borer. The PDMS substrate and glass coverplate were exposed to an air plasma (PDC-32G, Harrick Scientific Products, Inc., Pleasantville, NY) for 30 s and permanently joined.

Glass/Glass Devices. Glass microfluidic devices were fabricated using soda lime glass substrates coated with $120 \mathrm{~nm}$ of $\mathrm{Cr}$ and $530 \mathrm{~nm}$ of AZ1518 photoresist (Telic Co., Valencia, CA). Substrates were exposed through the same photomasks with an exposure energy of 200 $\mathrm{mJ} / \mathrm{cm}^{2}$. The exposed substrate was developed for $2 \mathrm{~min}$ in Microposit MF-319 Developer (Rohm and Haas Electronic Materials, Marlborough, MA) and rinsed with water (18 M $\Omega$-cm, SuperQ Plus, Millipore Corp., Billerica, MA). The channel pattern was transferred to the chromium film with an 8 min etch in Cr Etchant 8002-A (Transene Co., Inc., Danvers, MA). Buffered oxide etch (Transene Co., Inc.) was then used to etch the channel pattern into the glass 
substrate until the channels were $\sim 10 \mu \mathrm{m}$ deep. Holes for fluid access were drilled into the substrate at the ends of each channel with a sandblaster (AEC Air Eraser, Paasche Airbrush Co., Chicago, IL). The substrate was stripped of remaining photoresist with acetone and chromium with an 8 min etch in Cr Etchant 1020 (Transene Co., Inc.). The channels were $10.39 \pm 0.06 \mu \mathrm{m}$ deep, measured with the stylus profiler. Glass-to-glass bonding was used to seal the substrate with the channels to a soda lime coverplate. The substrate and coverplate were first sonicated in cleaning solution (Micro-90, International Products Corp., Burlington, NJ), rinsed with water, and hydrolyzed at $70^{\circ} \mathrm{C}$ for 15 min in ammonium hydroxide, hydrogen peroxide, and water (2:1:2). Following hydrolysis, the glass pieces were sonicated for $10 \mathrm{~min}$ in water. The patterned glass was clamped to the coverplate and heated at $90^{\circ} \mathrm{C}$ for $4 \min$ followed by a period of $3-4 \mathrm{~h}$ to drive off any remaining water. The bonded microfluidic devices were permanently annealed at $550^{\circ} \mathrm{C}$ for $10 \mathrm{~h}$. Glass reservoirs $(4 \mathrm{~mm}$ i.d.) were attached over each sandblasted hole with epoxy (353ND-T, Epoxy Technology, Billerica, MA). Prior to use, channels were flushed with 1 $\mathrm{M} \mathrm{NaOH}$ and water.

\section{Devices with One, Two, and Four Control Channels}

The basic microfluidic design used in this study is schematically depicted in Figure 1, and transmitted light images of the top of the sample transfer region for devices with one, two, and four control channels are shown in Figure S1. In the designs with two and four control channels, each set of control channels was combined into a single channel, which in turn was connected to

control reservoir A, B, C, or D. The distance between the 1D channel (or waste 2 channel) and the first control channel was $80 \mu \mathrm{m}$ center-to-center and all control channels were spaced 240 $\mu \mathrm{m}$ center-to-center. The sample confinement results from these devices are summarized in 
Figure 5 with four control channels providing the narrowest sample streams and largest concentration to width ratios.
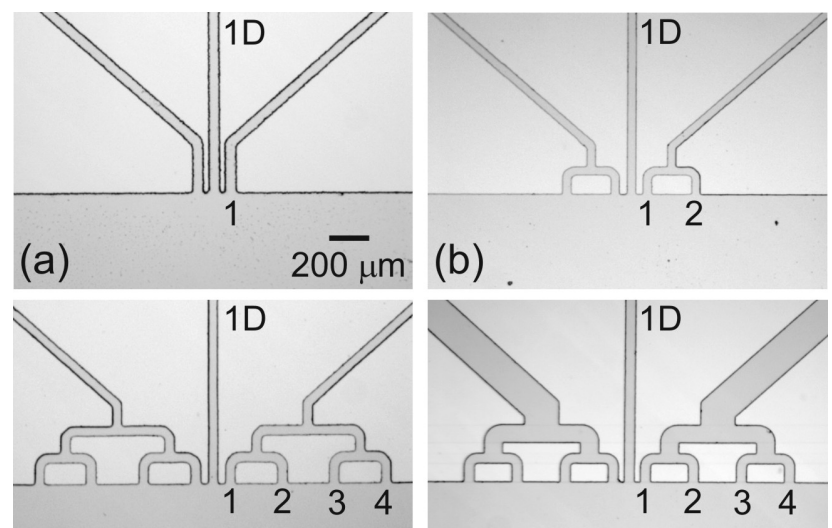

(c)

(d)

Figure S1. Transmitted light images of the top of the sample transfer region where (a) one, (b) two, and (c)-(d) four control channels join the 1D channel and planar region. The control channels are labeled 1,2,3, or 4, depending on the number in the design. The control channels in (d) were widened compared to the channels in (c) to minimize the potential drop between the reservoir and the sample transfer region. The $1 \mathrm{D}$ channel was spaced $80 \mu \mathrm{m}$ center-to-center from the adjacent control channel, and the control channels were spaced $240 \mu \mathrm{m}$ center-tocenter. Scale is the same in all images.

\section{Comparison of PDMS/Glass and Glass/Glass Devices}

To ensure that these sample transport schemes could be effectively transferred to glass devices, a series of confinement experiments similar to those shown in Figure $\mathbf{4}$ was performed using the glass microchips. Glass devices with the parallel channel design were fabricated using the same photomask used to create the SU-8 masters, but the channel dimensions were slightly wider because of the isotropic etching of the glass substrates. The results are shown in Figure S2 for fluorescein and rhodamine $\mathrm{B}$ and compared to the results from the PDMS/glass device. Rhodamine B has a slightly smaller diffusion coefficient and a higher electrokinetic mobility 
than fluorescein; however, the sample stream widths were comparable. Also, the performance of PDMS/glass and glass devices was similar, illustrating that the methods described thus far could be easily transferred between substrate materials without loss in performance. These results also confirmed that flow variations were not occurring in the hybrid PDMS/glass devices because the channel bottoms were glass and the other three walls were PDMS.

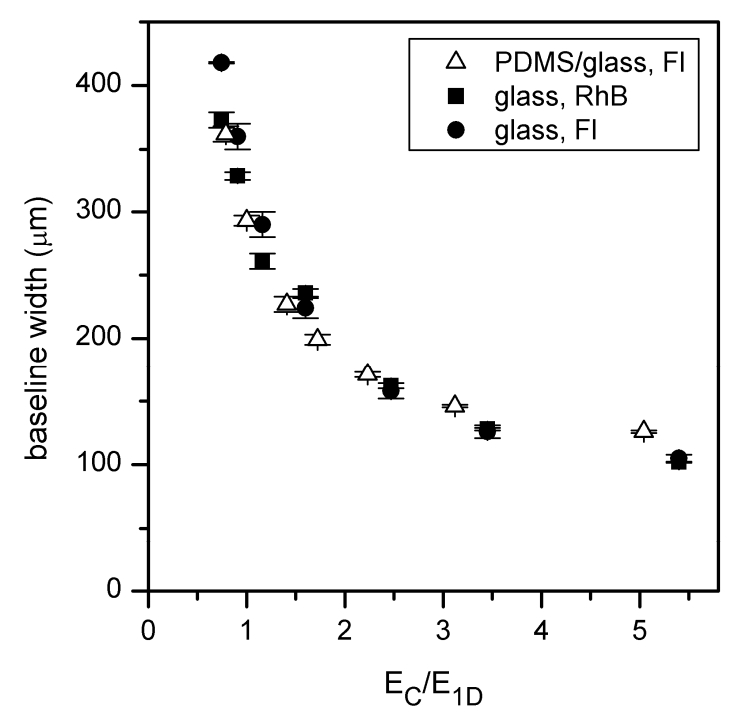

Figure S2. Variation of the sample stream width $(4 \sigma)$ with $\mathrm{E}_{C} / \mathrm{E}_{1 \mathrm{D}}$ ratio for a PDMS/glass device and a glass device. Samples were fluorescein (Fl) on the PDMS/glass chip and fluorescein and rhodamine $\mathrm{B}(\mathrm{RhB})$ on the glass chip.

\section{Injections into the Sample Transfer and Planar Regions}

As described in the experimental section, a gated valve was used to inject sample into the 1D channel for transport into the sample transfer region. Injections were evaluated on both the open and parallel channel designs. Similar to the sample stream experiments described above, the sample plug must travel through the sample transfer region without being distorted. The plug should not have one side moving faster than the other or assume a horseshoe shape. A series of images showing a sample plug being transported through the planar region on the parallel 
channel device is shown in Figure S3. These images were from a 200-ms injection with $E_{C} / E_{1 D}$ = 3.9. The sample moved directly across the sample transfer region, not deviating from the expected path and shape. The fluorescence signal was fitted to a Gaussian function along both axes. The plug width correlated well with the data for continuous sample stream measurements in the parallel channel design and fell where expected along the curve in Figure 6a.

In order to transport samples in two dimensions, there must be a simple and efficient manner to transfer sample. With these device designs, the applied potentials could be toggled to effect transport in either dimension. The first test was to look at how a sample front moved into and down the planar region. For the loading stage of this planar injection, a confined sample stream was allowed to flow into the sample transfer region, similar to Figure 4. Once the front was established, the potentials at the sample, waste 2, and control A-D reservoirs were removed, and $3 \mathrm{kV}$ was applied at the buffer 2 reservoir with the waste 3 reservoir grounded. The overall shape and movement of the sample front in the planar region were good (data not shown), however sample leaked into the sample transfer region from the 1D channel, and subsequently, into the control channels. To prevent sample from leaking out of the 1D channel and into the control channels, the applied potentials were adjusted as follows, and Figure S4 shows the injection of the sample front. A small potential was applied to the sample and waste 2 reservoirs to pull sample back from the sample transfer region, and simultaneously, a higher potential was applied to the control A and C reservoirs to push the sample front cleanly into the planar region. Control reservoirs B and D had no potential applied as before. As seen in Figure S4c, the sample did not flow out of the 1D channel after launching the sample front into the planar region. In Figure S4d, variation between plug lengths was determined to be less than 3\% RSD. The differences in position were likely due to small variations in channel dimensions. Even though band shape was 
quite good, there was still a small amount of sample leaking into the first control channels. Once discrete plugs were injected into the planar dimension, this was not as much of an issue.

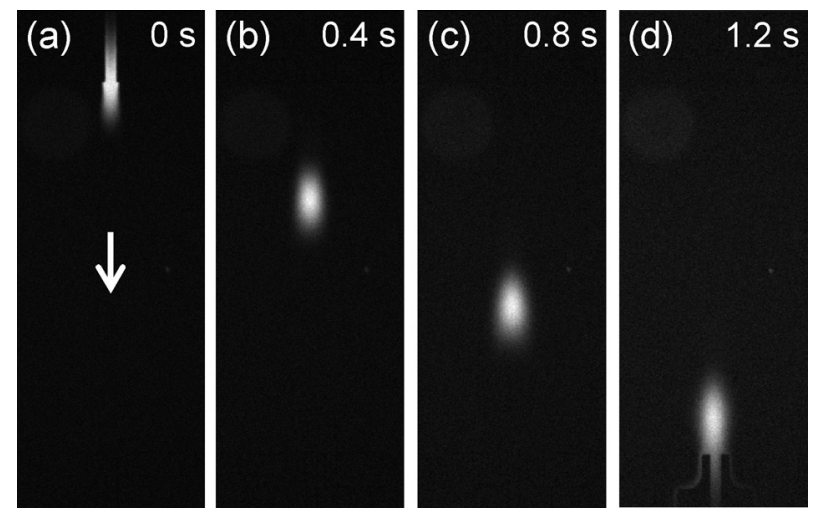

Figure S3. Fluorescence images of a sample plug transported through the sample transfer region for the parallel channel design at (a) 0 , (b) 0.4 , (c) 0.8 , and (d) $1.2 \mathrm{~s}$ after entering the sample transfer region. The gated injection time was $200 \mathrm{~ms}$, and $\mathrm{E}_{\mathrm{C}} / \mathrm{E}_{1 \mathrm{D}}=3.9$.
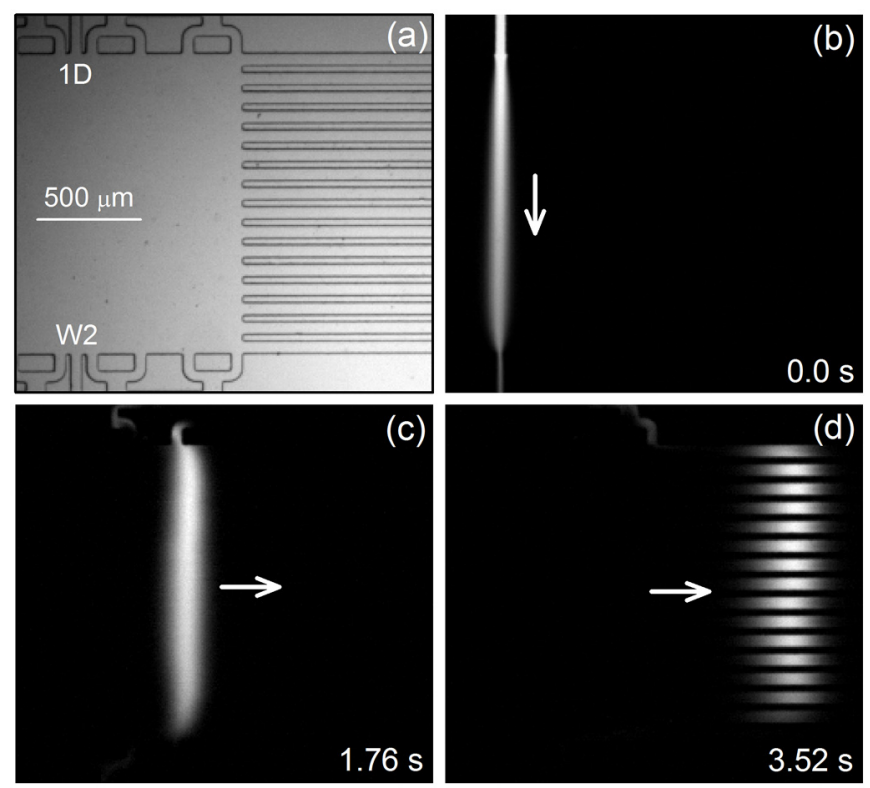

Figure S4. (a) Transmitted light image of the sample transfer region for the parallel channel design and fluorescence images of a sample front dispensed into the planar region at (b) 0 , (c) 1.76, and (d) $3.52 \mathrm{~s}$ after reconfiguring the potentials. Scale in (a) applies to all images. 


\section{References}

(1) Duffy, D.C.; McDonald, J.C.; Schueller, O.J.A.; Whitesides, G.M. Anal. Chem. 1998, 70, 4974-4984. 